\title{
Beyond notification: Filling gaps in peer production projects
}

Authors' preprint. Accepted for publication in New Media \& Society journal on 2 February 2018. Please consult journal edition when citing.

Suggested citation (awaiting edition number and page numbers):

Ford, H., Pensa, I., Devouard, F., Pucciarelli, M., Botturi, L. (2018) Beyond notification: Filling gaps in peer production projects. New Media \& Society. Sage Publishing.

Dr Heather Ford

The University of New South Wales

Dr Iolanda Pensa

SUPSI University of Applied Sciences and Arts of Southern Switzerland

Florence Devouard

SUPSI University of Applied Sciences and Arts of Southern Switzerland

Marta Pucciarelli

SUPSI University of Applied Sciences and Arts of Southern Switzerland

Dr. Luca Botturi

Scuola universitaria della Svizzera italiana

Acknowledgements:

We wish to thank the Wikipedians and academics involved in the project for their time and comments on the paper and to the reviewers who provided helpful suggestions on the draft versions.

With the support of Swiss National Science Foundation (SNSF) and the South African National Research Foundation (NRF) in the frame of the Swiss-South Africa Joint Research Programme (SSAJRP) for the research project "Wikipedia Primary School: Providing on Wikipedia the information necessary to complete the cycle of primary education in the languages used by the different education systems" (grant number IZLSZ1_148975).

\section{Introduction}

The Wikipedia Primary School Project was initiated in 2014 in order to stimulate the development of Wikipedia content in articles relating to the South African primary school curriculum. Over the next three years, a group of Wikipedians, academics and practitioners applied a number of strategies promoted by Wikipedians to improve almost 200 articles in the English Wikipedia corpus. Wikipedia content relating to topics such as the "San people" (indigenous, hunter-gatherer people from Southern Africa) were weak before the project 
started. Improving them would enable Wikipedia to be useful to by primary school teachers in a region where access to textbooks is difficult, and to increase the quality of Wikipedia more generally.

The team tested a series of interventions including online writing contests and face-toface workshops to teach new editors how to edit Wikipedia ("edit-a-thons") in order to stimulate the development of target articles. On the wiki itself, however, efforts first focused on notification strategies: lightweight mechanisms for alerting editors about the existence of gaps and the need to work on targeted articles relating to the project. We found, however, that notification strategies largely failed to engage interest from either the existing editing community or readers who might be turned into editors. Notification requires enlisting the help of already enrolled editors and is too "thin" a mechanism when a relationship of trust does not already exist (between new editors and seasoned editors, for example).

In response, we identified local academics who had expert knowledge of target articles but who were not experienced editors and facilitated a process in which they would provide an "expert review" of target articles. Wikipedia editors ("Wikipedians") then responded to reviews by making relevant changes to articles. Although not wildly successful by traditional measures, the expert review process elicited significantly more edits to articles than notification mechanisms alone and resulted in two articles moving to "featured article" status, where Wikipedians deem articles to be of the highest quality. Furthermore, the expert review process introduced a number of local experts to the Wikipedia editing process and therefore offers lessons for the important goal of filling gaps in peer production projects.

\section{Filling gaps}

The early history of the internet was characterised by celebrations of the expansive qualities of the network as the costs of producing and sharing information among geographically dispersed groups fell. The internet and its related affordances, as the story went, enabled anyone with access to express themselves creatively (Jenkins 2006), organise around common concerns (Benkler 2006, Shirky 2009) and provide products and services for a seemingly infinite inventory of niche users (Anderson 2006).

With nearly 500 million readers, 295 linguistic editions and more than 40 million articles, Wikipedia is the posterchild for what Benkler (2006) call "commons based peer production" in which large numbers of people work cooperatively to produce collective, public goods. The commons-based peer production model appears so successful that Wikipedia has been presented as the antidote to platform capitalism and neoliberalism (Fuchs 2014). According to these framings, Wikipedia is the antithesis of "the market" because it does not rely on managerial commands or advertising and because its content can be freely used, reused and modified for commercial and non-commercial purposes.

Whereas the networked communication environment has produced significant innovations, Wikipedia being but one example, the evolution or maintenance of such systems have demonstrated two key problems. Firstly, although peer production projects like Wikipedia and OpenStreetMaps have produced global public goods that rival their commercial counterparts in many respects, these projects are characterised by significant inequalities inequalities that tend to exacerbate existing asymmetries e.g., between developed and developing countries and between different genders.

Wikipedia suffers from a systemic bias problem: the project is dominated by the perspectives and cultures of white, Western males while women, the knowledges and cultures of the developing world and those of minority groups tends to be very weakly represented. Research has shown that Wikipedia's articles, topics and contributors are more likely to represent the United States and Western Europe with large parts of the developing world under-represented. Only about $1 \%$ of edits originate from Africa and countries, cities and towns in Africa are significantly under-represented (Graham et al. 2015). Similarly, research on the gender of Wikipedia editors indicate that about 85\% are male (Glott et al. 2010; Hill and Shaw 2013), that topics with a female audience are weakly represented (Lam et al. 2011) and that 
Wikipedia is more likely to have missing biographies on women than on men (Reagle and Rhue 2011).

The second key problem lies in the retention of volunteers. The number of active contributors to Wikipedia declined after it reached a peak in 2007. Although the decline may now have stabilized (Erhart and Halfaker 2015), research surrounding the reasons for editors leaving the project indicate significant bias against the needs, experiences and concerns of newcomers (Halfaker, Geiger, Morgan and Riedl 2013).

In the face of such challenges, a number of Wikipedia's stakeholders have attempted to increase the quality and quantity of Wikipedia's edits and editors - both in areas where Wikipedia is weak and in general terms. The dominant strategy for filling Wikipedia's gaps has been to develop notification systems that alert users to the existence of gaps. Notification systems were developed early on by the Wikipedia community as a method for leveraging the interests and knowledge of editors (Cosley et al. 2007) and in drawing attention to tasks that the community deemed important (Krieger et al. 2009). Notification mechanisms are useful because they can be easily applied at scale and their success can be easily and quickly gauged. Editors are typically notified about gaps that need working on via a number of working web pages where Wikipedians tend to gather. These include WikiProjects (groups of Wikipedia editors who self-organise to improve content around particular themes or tasks), competitions for wiki editing (such as the WikiCup) or via portals like Wikipedia's Community Portal that serves as a hub for learning about Wikipedia and working on various editing tasks (WarnckeWang et al. 2015).

Other interventions have focused on enrolling newcomers into editing the encyclopedia - often discussed as a conversion from readers into editors. Edit-a-thons are typically face-toface events in which seasoned Wikipedians train new users and/or work together on improving particular areas of the encyclopedia. Academic institutions have often played important roles as facilitators of such events - hosting the edit-a-thons, editing articles relating to academics and academic research, and facilitating events to fill particular gaps. Such events have been used to improve, e.g., Wikipedia's coverage of women (Lavin 2016), people of colour (Barnett 2013) and particular collections such as that of the British Library.

Another key intervention in which academics have played an important role is in facilitating the writing and editing of Wikipedia articles as part of classroom assignments (Farzan et al. 2016). Any university lecturer around the world could technically initiate such a project, but the Wiki Education Programme provides specialised support to universities in the United States and Canada through the Wiki Education Foundation, a non-profit organisation based in San Francisco. Academics are provided with support materials for teaching, sample syllabi, assignments and handouts. The programme also support Campus Ambassadors and Online Ambassadors who are able to teach students in the US and Canada how to edit Wikipedia and who answer students' questions online ${ }^{1}$.

Academics have also contributed to Wikipedia via processes for writing encyclopedia articles for peer reviewed academic journals which are then available to be re-incorporated into Wikipedia. This model has been used by the Public Library of Science (via the PLoS Computational Biology and PLoS Genetics journals) and the WikiJournal of Medicine. Articles are written in the style of a Wikipedia article by academics and then peer reviewed before being published in the journal. A copy of the article is then posted to Wikipedia where it continues to be updated according to Wikipedia's own editorial practices.

Other initiatives have sought to engage academics in the review of Wikipedia articles using a process called "expert review". Before our project started, expert reviews had been used to engage scientists and medical doctors in the improvement of particular sets of articles but had not yet been used to bring reviews into Wikipedia or been used in the context of unpaid reviews. Google.org's "Health Speaks" project enlisted the help of professional medical editors hired (and paid) by the Google Foundation in 2010 to review a series of health-related articles using Wikipedia's quality criteria.

\footnotetext{
${ }^{1}$ See https://en.wikipedia.org/wiki/Wikipedia:Education_program
} 
A large variety of interventions have, in summary, been tested to catalyse the editing of particular sets of articles - both by current editors and by enrolling new editors, particularly in the academic and education sectors. Some useful research has been done to test the success of such interventions, but the problem is that research tends to focus on counting edits made after a particular intervention and evaluating quality according to increases in the size of articles or other statistical methods (Halfaker 2017). Although such research provides general indications of what kinds of interventions result in larger quantities of edits over longer periods of time, there is little understanding of why certain interventions succeed where others fail in particular contexts. In order to develop the most appropriate interventions, we needed a much better understanding of Wikipedia articles as representing the knowledge of particular assemblages of actors. The identities, expertise and authority of actors and the relations between them affects the outcomes of knowledge work and deserve special attention.

\section{The Case}

The Wikipedia Primary School Project was aimed primarily at primary school teachers who use Wikipedia articles to inform their own teaching, or to adapt articles and assets (such as images) associated with those articles for use by students in the classroom. The premise was that, if teachers had access to high quality encyclopedic content relating to the South African national curriculum on Wikipedia, then they would more likely be able to build well-resourced lessons using this free resource.

South African primary schools still face major challenges including a lack of adequate infrastructure, books and libraries to support learners, low teacher to student ratios and high levels of teacher absenteeism (Bernstein 2011). In 2015, 57 million South African children of primary school age were still out of school (UNESCO 2015) and South African primary schools were placed 132 out of 144 countries with regard to quality teaching (WEF 2013). Although it has been reported that only $6 \%$ of classrooms have computers connected to the Internet (Isaacs 2007), access to the internet via mobile phones is significantly higher and Wikipedia is the sixth most visited website in the country (Alexa 2017). All but one of South Africa's eleven official languages has a Wikipedia edition ${ }^{2}$ and a number of projects initiated by volunteers and supported by Wikimedia Chapters and User Groups have been developed to make Wikipedia accessible offline.

Our study adopts a canonical action research approach that applies Davison, Martinsons \& Kock's (2004) principles for ensuring research rigor and relevance in action research. Action research involves "solving organizational problems through intervention while at the same time contributing to knowledge" (Davison et al. 2004, p65). In this case, solutions to Wikipedia's systemic bias were developed and tested, and the results reflected upon in order to inform future interventions and studies.

The cyclical process involved a thorough diagnoses of the problem (initiated during a feasibility study prior to this project) followed by planning, intervention and reflection using an iterative, cyclical approach. Planned actions were designed to specifically address the observed problem and its causes (the principle of change through action) but flexibility in the plan ensured that the project team could adapt interventions to emerging or changed circumstances (the principle of learning through reflection). Such changes are demonstrated in the need to adapt strategies to involve academics in the editing process as discussed further in the paper.

A scientific committee comprising of twenty South African and international stakeholders was established to guide the project. Experts included scholars and researchers selected for their expertise in the field of ICT and education, primary school education, Wikipedia experts, and teachers and parents from two local primary schools.

Members of the committee were responsible for defining a list of articles relevant to the South African national primary school curriculum and providing feedback on the project as it evolved. The establishment of the scientific committee ensured that the project remained rooted

\footnotetext{
${ }^{2}$ Southern Ndebele has not moved from the incubator to official Wikipedia edition status.
} 
on the problems experienced at a local level and that the project team remained accountable to its stakeholders. The project team kept all stakeholders informed of the project's progress through the iterative updating of a number of project webpages on Wikimedia's "meta" site that is used by Wikimedians to document and share information related to organisational and project development issues ${ }^{3}$. The project was theoretically grounded by a close reading of relevant literature and related studies.

A set of 183 articles from English Wikipedia ${ }^{4}$ were selected by the committee at the start of the project. Subjects ranged from the social sciences and life orientation to natural sciences. Social science-related topics included history and geography, life orientation included subjects relating to health, hygiene and nutrition, and natural science-related topics ranged from biology to ecology. Topics were either South Africa specific (e.g. "farming in South Africa" or "South African cuisine"), pan-Africanist (e.g. "African musical instruments") or generalist (e.g. "wood", "pollution" and "boiling"). A number of social issues were also identified by the group to include topics relating to bullying, stereotyping and bias, child abuse, alcoholism and domestic violence.

A sample of topics related to the "Land Transport" subject area of the national curriculum can be found in table 1 below. In this example, six topic areas from the national curriculum were matched with nine articles on English Wikipedia, four of which were specifically South Africa related. The South African-related Wikipedia articles are shaded in the table below.

Table 1: Topics and articles in the 'Land Transport' subject area

\begin{tabular}{|l|l|}
\hline Topic from the curriculum & Related English Wikipedia article titles \\
\hline Animals & Animal powered transport \\
\hline Carts, wagons, coaches & Transport in South Africa \\
\hline \multirow{2}{*}{ Bicycle } & Bicycle \\
\hline \multirow{2}{*}{ Steam engines and trains } & Rail transport \\
\cline { 2 - 2 } & Rail transport in South Africa \\
\cline { 2 - 2 } & South African Locomotive history \\
\cline { 2 - 2 } & Two foot gauge railways in South Africa \\
\hline Motor cars & Automobile \\
\hline $\begin{array}{l}\text { Common forms of transport for people } \\
\text { and goods today }\end{array}$ & Transport \\
\hline
\end{tabular}

After articles were identified, the project team set about trying to catalyse their improvement using a number of existing strategies highlighted in the literature review. Strategies included notifying editors about the need to develop target articles directly on Wikipedia ("notifications"), contacting local academics and other subject-matter experts to review articles ("expert reviews") and encouraging local and regional academic journal editors to adopt the PLoS model for creating Wikipedia-style journal articles that can then be moved to

\footnotetext{
${ }^{3}$ See https://meta.wikimedia.org/wiki/Wikipedia Primary School

${ }^{4}$ English Wikipedia was selected as the target for improvement efforts, both because of the breadth and depth of the database and the higher contribution rate necessary for maintaining articles. Although Wikipedia exists in ten of the eleven official languages of South Africa, most of the other language editions are limited by low contribution and access rates.
} 
Wikipedia ("topic pages"). We also used target articles as subjects for local edit-a-thons and editing competitions.

Editing competitions proved relatively successful intervention, although this failed to bring newcomers into the editing process and some articles (particularly those with few online sources and reliant on highly specialised knowledge) were still not developed using this method. No articles were improved through the local edit-a-thons.

Notification strategies proved largely unsuccessful. Commonly used strategies such as calls for participation, calls for article creation, and article assessment on WikiProject talk pages had extremely low impact or no impact at all. The majority of notifications were delivered via WikiProjects. WikiProjects are portals on Wikipedia where groups of Wikipedia editors coordinate editing activity according to themes of work (e.g. WikiProject South Africa). The typical WikiProject consists of a few participants monitoring and maintaining a project communication hub that supports a wide variety of work activities involving a large number of peripheral participants (Morgan, Gilbert, McDonald \& Zachry 2013).

Most of our requests to WikiProjects were simply not considered by any editors even though the WikiProject had not been tagged as inactive. In some cases, our request was disregarded while the automated log showed regular reassessments of other WikiProject articles. This suggests that although some WikiProjects communities are still active and are regularly reassessing articles, the assessment is being performed according to individual editor interest without a coordinated effort to follow up with any of the requests that are posted. Further investigation showed that whilst some WikiProjects are still active, most are now either dormant or extinct. WikiProjects were set up in the 2007-2008, as part of a global effort by the community, to prepare a print version of Wikipedia. In August 2016, however, only 37 WikiProjects from a total of 2466 (1.5\%) showed more than 1000 human edits to its pages over the entirety of the previous year.

In terms of outreach to academics, academic journal editors proved largely unresponsive to our suggestions for adopting the "topic pages" model. Only two editors responded positively but did not respond to subsequent correspondence. Negative responses included a response from an editor who wrote that they wouldn't participate because they did not recognise Wikipedia as a legitimate academic enterprise: "We are not interested in contributing to Wikipedia with its superficial coverage of topics and unacademic analysis of research findings let alone evidence-based." (17 March 2016)

The process that we followed for eliciting and responding to expert reviews of our target articles demonstrated the most sustained engagement with the highest quality results of all our interventions. Although overall numbers of articles that were improved as a result of this intervention was quite low 5 , there were two key elements of success involving both the high quality of articles produced as a result of the expert review and the positive interactions between academics and Wikipedians that resulted from successful examples.

\section{Expert reviews}

The expert review process was designed around a series of key steps. First, local academic experts were located through personal contacts of the project committee and according to online searches. Experts were asked via email to review articles specifically related to their areas of expertise. After an expert had responded positively to the requests to review an article, Wikipedia editors would be notified of the expert's involvement on the talk page of the article. This notification included the name of the expert, their affiliation, and a link to their personal webpage. Once an academic expert was assigned to an article, the Wikipedia community would be given two weeks to perform any final edits and additions before the article was sent (as a PDF in an email) to the expert. Finally, the expert reviews would be sent back to the team who then uploaded them to Wikimedia Commons and linked them to the talk page of the related article, where editors would be requested to respond by making edits to the article

\footnotetext{
${ }^{5}$ About a quarter (32 out of 136) academic experts who we contacted responded positively to requests.
} 
in response to suggestions from the academic expert. In each case, reviews might also involve edits as Wikipedians make changes to get the article "review ready" and when academic experts make suggestions to the article PDF via email to the project team.

When experts responded positively to requests, Wikipedia editors were notified about the expert's involvement with a request to get the article ready for expert evaluation in two weeks' time. Academic experts were then emailed a document template listing a series of questions for evaluating the specified article in addition to a PDF of the article. Figure 1 illustrates the first section of the template which links to Wikipedia's criteria for featured articles, provides spaces for reviewers to add their name and institutional affiliation, and confirm that their text be made available under Creative Commons open content licenses.

Questions in the template accorded generally with Wikipedia's own criteria for quality assessment. Quality assessment on Wikipedia is performed using a scale, with "stub" at the low end indicating a very basic description of the topic to "FA" (Featured Article) status, the highest quality level accorded to Wikipedia articles by passing an official review by a central Wikipedia body. Assessments are performed on a voluntary basis with varying levels of discussion and consensus. Grades are not static and may be challenged over time. Featured articles, according to Wikipedia's criteria, are characterised by "professional standards of writing, presentation, and sourcing" (Wikipedia n.d.). In addition to criteria relating to writing style, consistency and structure, featured articles must meet Wikipedia's policy requirements: they must be written according to Wikipedia's policy on neutral point of view, all content must be compliant with Wikipedia's open content copyright standards and all claims verifiable against reliable sources.

Reviewers were asked to comment on the quality of the summary, the structure and style of the article and the references, for example. Reviewers were asked whether "the summary of the article a complete, thorough, and concise introduction to the topic", whether there are areas for improvement, whether "meaningful data (were) missing" and whether there was anything "too detailed for a general overview of the topic". In addition, reviewers were asked whether the article was "representative of the international dimension" of the topic and whether it featured examples from around the world. This criterion is not explicitly included in Wikipedia's quality assessment criteria but is obviously important for the purposes of this project.

The system was improved in the second stage of the project. Expert reviews were initially published as a PDF document on Wikimedia Commons and linked to the article's talk page but it became clear that responding to suggestions in a remote PDF was more difficult for editors than it needed to be. As a result, expert comments from the review were directly copied onto the article talk page. Editors could then check off each item as they made relevant changes to the article as can be seen in figure 1. 
Figure 1: Talk page of the 'Herero and Namaqua genocide' on English Wikipedia

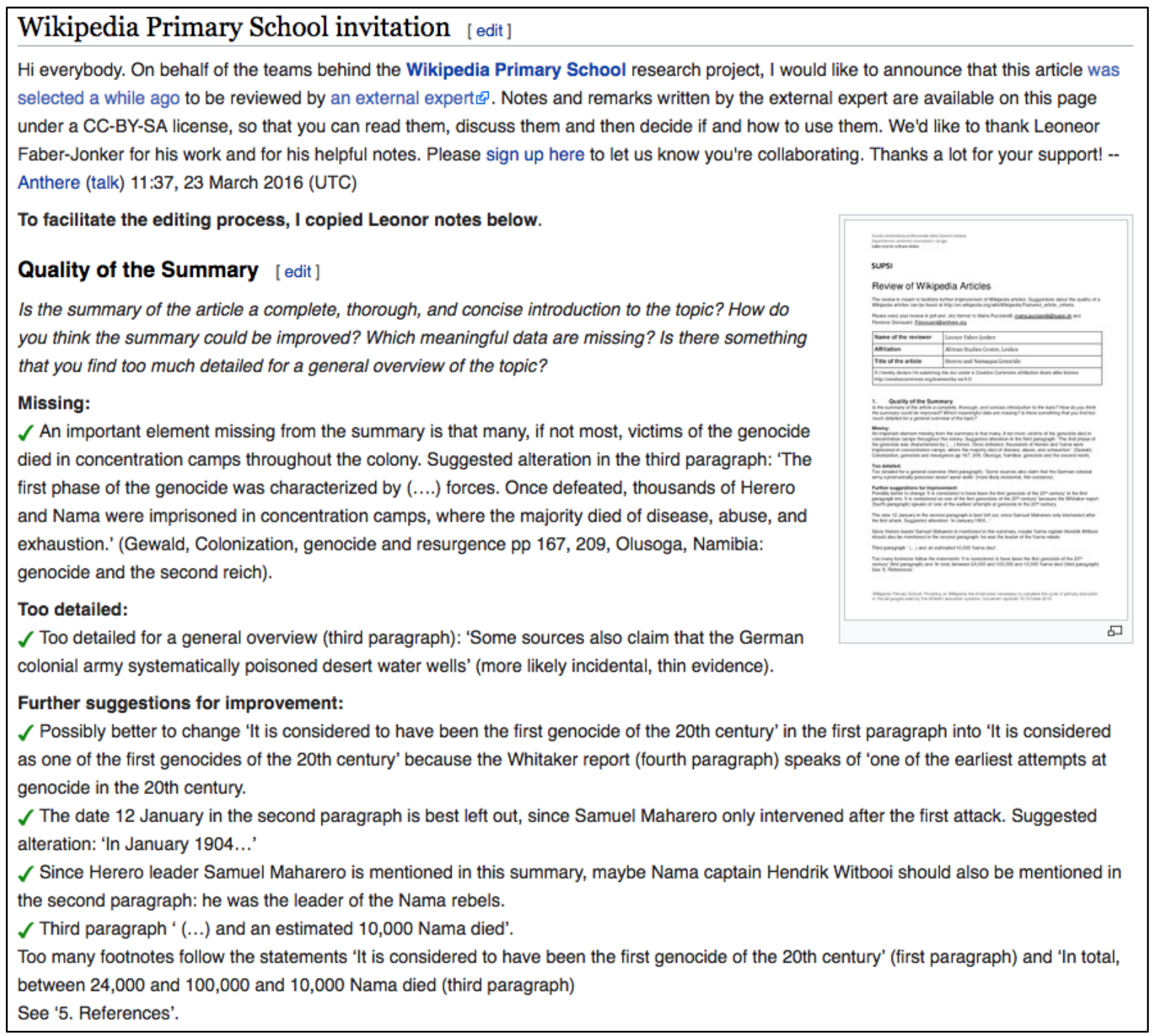

In order to correctly attribute the reviewers' comments and to verify that academic experts had granted permission to have their comments licensed under Creative Commons open content licenses, the project team made use of Wikimedia's OTRS (Open Source Ticket Request System) system. OTRS is the software used by Wikimedia projects to handle queries, complaints, statements, and comments from the public by email. Despite the fact that the author and license are provided when content is uploaded, Wikimedians have determined that they require confirmation that the copyright holder of images or text provided on Wikipedia or Wikimedia Commons has, in fact, granted permission for their materials to be used under Wikipedia's open content licensing terms.

After uploading the review, a template email stating the authorship and license information of the content was sent to the expert. The expert was asked to send this email himself/herself to the OTRS permission system for archival purposes. OTRS agents acknowledge receipt of the appropriate documentation to confirm authorship and licence of the document. The existence of the record was then mentioned on the article talk page by the OTRS agent.

The majority of academic experts chose not to edit the article directly on Wikipedia but rather to fill out the expert review template. Some reviewers strictly followed the template, but provided general feedback rather than listing specific items for editing or improvement. In other cases, reviewers left aside the generic template and made comments on the PDF of the 
article itself using Adobe's commenting functionality while the final approach by reviewers involved an iterative review of each line or paragraph of the article. Once expert reviews had been published to article talk pages, Wikipedia editors responded by editing and/or responding through textual notes to specific concerns of expert reviewers on the talk pages.

\section{Discussion}

Two key learnings from the project were that notification mechanisms, alone, will not result in gaps being filled, and that iterative and sustained engagement with outsiders (in this case academics) was necessary to developing high quality articles and potentially longer term positive relationships of academics to Wikipedia. The question remains, however, why some interventions were more successful than others.

Star and Greisemer's work on "boundary objects" is a useful starting point for understanding why some interventions fail where others succeed, and indeed, in thinking about new metrics for success in this area. The theory of boundary objects was originally developed by Star and Griesemer in 1989 and developed by Star in subsequent publications (e.g. Bowker and Star 2000, Star 2010). Studying the Museum of Vertebrate Zoology at the University of California, Berkeley, Star and Griesemer tried to understand how the museum was able to build up its research collection of vertebrates through the help of amateur naturalists and other stakeholders in both the lay and academic worlds. They found that the museum director, Joseph Grinnell was able to enrol a diverse set of actors in helping to develop the collection through an elaborate system of curation guidelines.

Those guidelines constitute boundary objects defined as:

"objects which are both plastic enough to adapt to local needs and the constraints of the several parties employing them, yet robust enough to maintain a common identity across sites. They are weakly structured in common use, and become strongly structured in individual-site use. These objects may be abstract or concrete. They have different meanings in different social worlds but their structure is common enough to more than one world to make them recognisable, a means of translation." (Star and Griesemer 1989, 393)

Wikipedia's goal of building "the sum of all human knowledge" can be likened to Grinnell's task of collecting examples of all vertebrates in California. Wikipedia needs to enrol allies in helping to build the encyclopedia. Academics are an important ally for Wikipedia: some academics already engage in projects that attempt to fill Wikipedia's gaps through edit-a-thons and online experiments, and there have been productive results from using Wikipedia editing to achieve the academy's pedagogical goals through the Wiki Education Programme.

The problems are that knowledge has different meanings for Wikipedians and for institutional academics that is difficult for many academics to overcome. Wikipedia has a policy of not publishing "original research", for example, and there have been high profile cases in which academic researchers have attempted to edit Wikipedia to include their new findings and been met with significant resistance (Messer-Kruser 2012). Furthermore, translating between different worlds is difficult given that credit does not always travel ${ }^{6}$, and technical expertise necessary for understanding how to represent knowledge within the rules, standards and practice is significant.

Although there are many similarities between Grinnell's museum and Wikipedia's database, there are important differences which add to the difficulties of enrolling allies. First, Wikipedia is an online encyclopedic project characterised by diffuse and inconsistent identities

\footnotetext{
${ }^{6}$ Some research indicates that citations to academic articles on Wikipedia result in an increase to citations in academic texts, but this finding is disputed by others (Sugimoto, Work, Larivière \& Haustein 2017).
} 
(editors can produce multiple user profiles which are often disconnected from real names) and Wikipedia lacks the important trust-building function of face-to-face engagements that Grinnell's museum enjoyed.

Despite this, some academics have engaged with Wikipedia and there are clear incentives for doing so: academics' research findings can be disseminated further than traditional academic publishing allows, academics who are cited on Wikipedia may benefit from larger citation numbers in academic literature, and Wikipedia has been shown to be a good venue for developing real-world knowledge of students. Such "Wikipedian academics", however, tend to be outliers. As Brown and Duguid (2001) note, incentives are generally used to explain why certain results fail or succeed but individual motivation is unlikely to achieve the kinds of organizational coordination required to enlist outsiders - in this case in the work of maintaining and growing the encyclopedia.

In order for the two groups of experts (Wikipedians and academic experts) to work together, they must engage in negotiation through boundary work. Boundary objects are not, in themselves, good or bad at enabling coordinated work. Rather, there are particular elements of a successful negotiation in the process of developing boundary objects that make that object appear to be either successful or unsuccessful at achieving cooperation. Facilitating negotiation using such objects requires three key characteristics: a mutual recognition of the authority and expertise of both groups, a respect for different practices by which different actors create knowledge and, finally, a commitment towards iterative, interpersonal communication between different communities of practice in order to define and pursue shared goals.

\section{Recognising authority and expertise}

Boundary objects are successful when they "maximize both the autonomy and communication between worlds" (Star and Griesemer 1989,404). Unlike the journal editors who were asked to contribute using the "topic pages" model, the expert review process enabled the autonomy of both worlds to be maximised. Wikipedians did not oppose the authority of the expert academic reviewer when the review was published to the talk page, and academics recognised the expertise of Wikipedia editors to improve the articles based on their comments. The particularities of the communications between academics and Wikipedians was critical in establishing this mutual trust. When email requests were made to individual academic experts, facilitators demonstrated that we were aware of academics' particular areas of expertise when we requested an evaluation of a particular article or articles (rather than a generic request). Furthermore, the request was for the academic to evaluate (rather than to edit) the article. Asking for the expert's opinion in this way served to reinforce the academic's authority.

In turn, academics who assisted with reviews recognised the authority of Wikipedia as an everyday reference source for students and the public. When expert reviews were published, Wikipedians did not object to their placement. In most cases, editors moved through the suggestions and critiques in an iterative fashion. The Nelson Mandela article, for example, was reviewed by Dr Steven Friedman, a South African academic and public intellectual who directs a university research centre. In making an edit in response to a review of the Nelson Mandela article, Wikipedia user Zaian left the comment "per Steven Friedman's external review (see PDF linked from talk page), Harry Schwarz is not notable enough for inclusion here." This statement indicates that user Zaian recognises the authority of Friedman as an expert to make such a judgement.

Not all comments from the expert review were implemented by editors. Whereas Friedman argued in his review that "AB Xuma was not removed from the ANC Presidency by a vote of no confidence", for example, Wikipedia user Zaian responded that Sampson's biography claims he was. Another comment from Friedman that he could "find no reference anywhere to the person 'Noengland'" Zaian responded that he had found "a few references online to Noengland as Dalindyebo's wife". In such cases, Wikipedians were critically assessing the comments by the reviewer in relation to their reliable sources policies. Whereas academics can make claims in their field based on analysis of primary sources, Wikipedia policy indicates that 
editing must not rely on primary research and can only be based on what is published in a reliable source.

Although academics' suggestions were not accepted wholesale there was therefore still a successful translation of credentials and authority from academia to Wikipedia.

\section{Respecting work practice}

Boundary objects need to have just the right amount of plasticity and robustness to enable work to be done by groups who are used to different practices. It is significant that the majority of academic authors involved in the project chose to use the template provided in email correspondence to develop their content rather than editing on wiki. Whereas the majority of projects attempting to improve contribution to Wikipedia focus on getting experts to edit on wiki or evaluating the success of such strategies, this project demonstrates the importance of enabling experts to use their own tools, methods and timings to contribute. As we have learned, the practice of editing Wikipedia requires both a significant investment in time and a different approach to knowledge. Expert reviews were most successful when the project team was able to facilitate the knowledge development process as a go-between, translating the practices of Wikipedians and academics to one another.

The first aspect of practice involves timing. Academics involvement strongly depended on the time period when requests were made. A greater number of experts agreed to help at the beginning of the academic semester (February/March and September/October) rather than at the end. Whereas academics were given a month to conduct their evaluations, Wikipedians were only given a few weeks, thus accommodating the different time scales in which things happen in the different communities of practice.

The second aspect of practice involved format. Wikipedians tended to respond to expert reviews when those reviews were in a format that suited the iterative style of Wikipedia editing, rather than generic comments that required wholesale changes to an article. Where reviewers used the review template provided, there was significantly more success when comments involved an iterative review of each line or paragraph of the article, rather than more general feedback which was not detailed enough for the Wikipedia editors to respond to. Line-by-line reviews suited Wikipedia's modular and iterative work process. Each comment could be copied and pasted in the talk page and editors could check off each item once it had been edited in response.

Figure 2 (below) illustrates how Wikipedia user, Midnightblueowl listed Friedman's comments from the PDF review in the talk page of the article, responding to each of the issues on the talk page after editing the article and checking items off as he did so. In the first comment, Friedman notes that the article incorrectly states as a fact that Mandela served on the Central Committee of the South African Communist Party. Midnightblueowl responds that the article has now been edited to reflect Friedman's comment, noting that it is the SACP's claim that Mandela sat on its Central Committee. The claim has also been moved from the lead to the body of article in order to deprioritize the statement. In another example, Wikipedia user Zaian removed Harry Schwarz from the list of people who Mandela befriended when he started studying law at the University of the Witwatersrand.

Figure 2: Discussion of expert reviewer's comment on Nelson Mandela talk page 


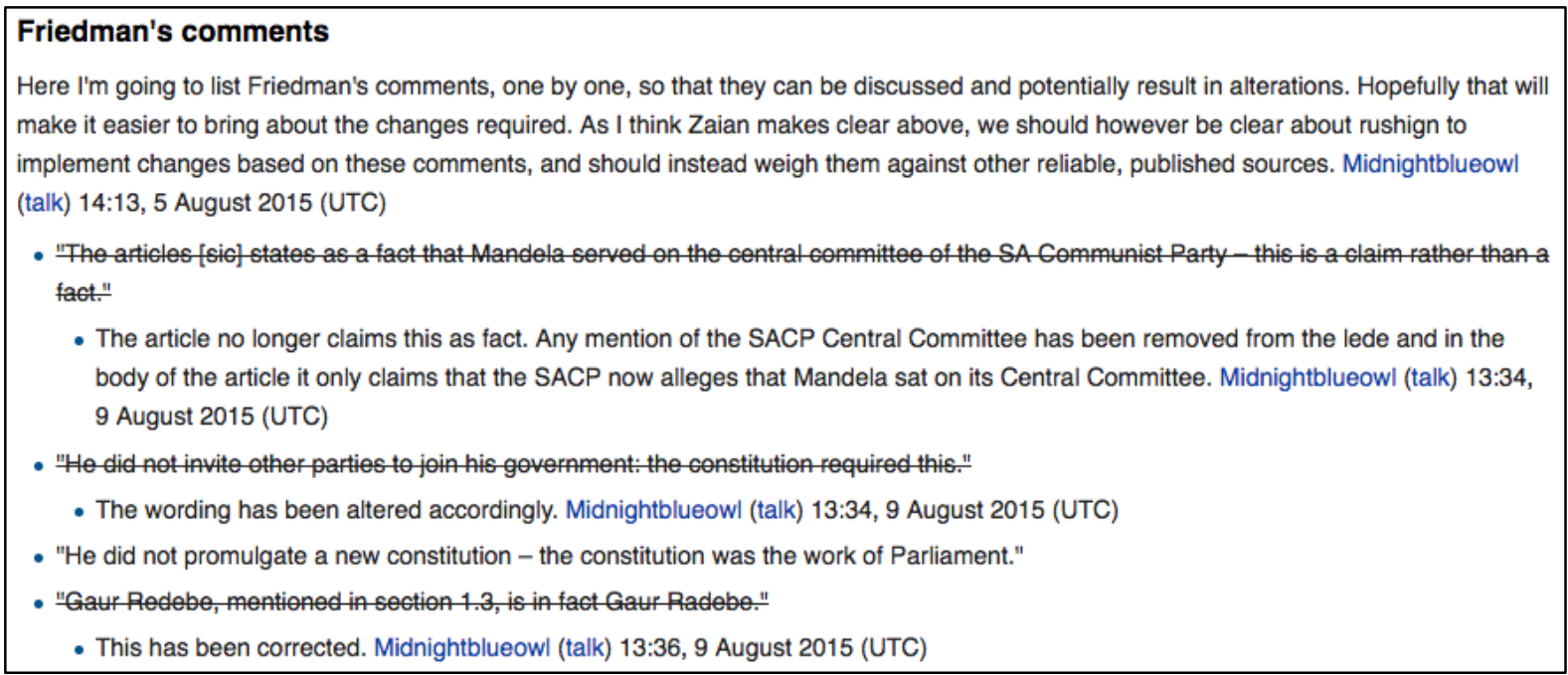

General feedback such as "the article is rambling and difficult to follow" may have been completely legitimate criticisms but tended to be difficult to respond to using Wikipedia's iterative approach. The format of comments also made a difference to their success when Wikipedia's editors responded to reviews. In some cases, reviewers left aside the generic template and made comments on the PDF of the article itself using Adobe's commenting functionality. The problem with such reviews was that PDF comments aren't always visible and navigating between the PDF and the article proved too difficult and/or time consuming for editors. The format of contributions, in other words, was a critical ingredient in successful coordination between Wikipedians and academics.

The third aspect of practice involved rules and policies, and it was in this aspect that translation of academics' contributions to Wikipedia's platform was less successful. Wikipedia requires that academic authors verify the authorship of their reviews published on Wikimedia Commons and that they agree to the Creative Commons license being applied to it. The OTRS process used by Wikipedia to manage this process proved cumbersome, time consuming and overly bureaucratic, especially since OTRS agents tended to apply different rules when assessing requests. This became a barrier to participation that frustrates simple translation efforts between outsiders and Wikipedia so that some potential participants had their contributions blocked from inclusion.

\section{Enhancing interpersonal communication}

Unlike the majority of interventions aimed at improving contributions to Wikipedia by particular groups, this project was characterised by an iterative approach in which strategies needed to be continuously adapted in response to the needs and concerns of its stakeholders. The process of refining the expert review required interpersonal communication between different stakeholder groups in which the feedback (both negative and positive) was taken seriously and procedures adapted accordingly. Neither the experimental method (where software is adapted and resulting online behaviour is evaluated through data collection) nor the method of evaluating contributions on wiki alone (where interventions focus on ways of encouraging or teaching users how to edit Wikipedia) would have resulted in the same success. Existing methods used to fill Wikipedia's gaps simply did not work on this occasion, and only through communication with potential contributors and changing strategies did articles develop according to the goals of the project.

Communication in geographically dispersed environments is certainly difficult and can result in the failure of boundary work. Sapsed and Salter (2004), for example, find that in geographically dispersed programs where there is no opportunity for face-to-face interaction, and/or ambiguous lines of authority, the project management tools under study were 
ineffectual as boundary objects. They argue that "a boundary object stands or falls on its capacity to accommodate local 'dialects'” (Sapsed and Salter, 2004, 1519).

Accommodating dialects in this project demanded flexibility of contribution (academics were given the option of editing directly on wiki or by using the template provided), adaptation to responses (Wikipedia editors tended not to respond to expert reviews when they were delivered in PDF format) and abandoning strategies where very little response was garnered. Digital communication enables a variety of potential contributors to be identified via their online academic profiles and enlisted via email. Communication via such mass strategies is useful for enlisting potential partners but continued interaction is essential to enable negotiation among actors. Indeed, even for enlisting partners, suggestions from the project committee and initial stakeholders were most successful in garnering contributors for the project as opposed to desktop research alone. As Marheineke et al. (2016) argue, boundary objects are only effective when they align the requirements of the social and technical dimensions to the collaboration process.

\section{Conclusion}

A number of interventions for filling Wikipedia's gaps particularly in subjects relevant to South Africa's primary school curriculum over the three years of the project's duration, changing course when interventions were unsuccessful and iterating on forms that demonstrated some success in enrolling new allies. Three key conclusions were reached based on our learnings from this project: the limits of notification as a method for filling gaps in peer production projects, the necessity of enrolling new groups who hold the knowledge that needs to be added to Wikipedia, and the importance of trust and negotiation in enrolling those groups.

First, our study reiterated recent findings (Warncke-Wang, Ayukaev, Hecht, \& Terveen 2015 ) that demonstrate the limits of notification as a method for filling gaps in peer production projects but produced new insight on why this may be the case. The majority of WikiProjects no longer fulfil the goal of coordinating editing tasks and so notification cannot catalyse the filling of prioritised tasks on Wikipedia. Perhaps most importantly, however, is the fact that notification engages groups who are already enrolled in Wikipedia and cannot catalyse editing activity outside of existing editors' interest or expertise.

Following from this, our second key conclusion is that enrolment of new groups is necessary to fill Wikipedia's gaps. Although local academics are a potentially valuable ally for Wikipedia, the problem is that academic editors may not have the time and tenacity necessary to overcome Wikipedia's steep learning curve. There is still a deep distrust of Wikipedia among academics as we demonstrated with the academic journal editors who responded to our requests for partnership by denigrating Wikipedia's authority. Although there are clear incentives for academics to engage with Wikipedia, incentives alone do not result in the kind of coordinated work that needs to happen in order to achieve the work of maintaining and growing the encyclopedia. Trust-building activities are essential for long-term engagement with academics.

Third, and finally, we learned that trust between Wikipedia editors and academics can only be garnered through facilitation in the creation of shared boundary objects such as the expert review that we develop here. Facilitation work that enables negotiation between editors and experts tends to be invisible work, but such facilitators are present in a number of Wikipedia projects - from "Wikipedians-in-residence" at museums and archives who facilitate the digitisation of those collections for use on Wikipedia to the "Campus" and "Online Ambassadors" who are able to teach students how to edit Wikipedia and who answer students' questions online as part of the Wiki Education programme. Active facilitation between academics and Wikipedians in this project was essential to developing the expert review as a boundary object able to "(live) in multiple social worlds and (have) different identities in each" (Star and Griesemer 1989, 409).

In summary, bringing in academics who have not yet engaged in Wikipedia editing (either directly in editing areas of their expertise or using Wikipedia in class assignments) 
requires negotiation, not merely notification. Whereas notification relies on single announcements to already engaged and literate members of the Wikipedia community, negotiation requires long-term engagement that iterates upon work practices that benefit and are conducive to both outsiders (in this case academics) and Wikipedia editors. Whereas notification requires outsiders to edit on-wiki, negotiation allows for each group to work on a common project (the article) separately. Notification strategies are evaluated according to whether they result in an immediate response by editors, negotiation strategies must be evaluated according to the long-term productive relationships that develop between expert communities. Instead of viewing potential editors as individuals with incentives, this project came to understand outsiders as individuals belonging to communities of practice who will only be successfully enrolled in Wikipedia editing through negotiation.

Wikipedia can become the site of successful boundary work but that this requires negotiation between Wikipedia experts and academic experts, a process that cannot easily be achieved through software mediated processes alone and a process that requires new measures for success that are based on relationships and long-term engagement. Successful boundary work requires the mutual recognition of the authority, respecting and recognising work practices and developing flexible mechanisms for communication between these different communities of practice. In particular, we demonstrate success when experts are able to contribute to Wikipedia using practices, standards, tools, timings and epistemologies that are familiar to them, rather than compelling them to edit on wiki.

Further work to refine boundary objects such as the expert review and the communication processes that support it are essential to understanding how Wikipedia reflects contemporary knowledge work and for how it could be used to develop a more equitable, diverse representation of the world's knowledges.

\section{References}

Alexa. 2017. "Top Sites in South Africa". Accessed September 18, 2017. https://www.alexa.com/topsites/countries/ZA

Anderson, Chris. 2006. The long tail: Why the future of business is selling less of more. Hachette Books.

Barnet, Fiona. 2013. “\#tooFEW - Feminists Engage Wikipedia”. Hastac, March 11, 2013. https://www.hastac.org/blogs/fionab/2013/03/11/toofew-feminists-engagewikipedia Accessed October 26, 2017.

Benkler, Yochai. 2006. The wealth of networks: How social production transforms markets and freedom. Yale University Press.

Bernstein, Ann (ed.). "Johannesburg: The Centre for Development and Enterprise." 2011. Accessed September 12, 2017. https://cdn.mg.co.za/uploads/2011/09/21/value-inthe-classroom-full-report.pdf

Bowker, Geoffrey C., and Susan Leigh Star. Sorting things out: Classification and its consequences. MIT press, 2000.

Brown, John Seely, and Paul Duguid. 2001. "Knowledge and organization: A social-practice perspective". Organization science 12(2): 198-213.

Cosley, Dan, Dan Frankowski, Loren Terveen and John Riedl. 2007. "SuggestBot: using intelligent task routing to help people find work in Wikipedia". In Proceedings of the 12th international conference on Intelligent user interfaces:32-41. New York: ACM 2007.

Davison, Robert, Maris G. Martinsons, and Ned Kock. 2004. "Principles of canonical action research." Information systems journal 14 (1): 65-86.

Farzan, Rosta, Saiph Savage, and Claudia Flores Saviaga. 2016. "Bring on Board New Enthusiasts! A Case Study of Impact of Wikipedia Art + Feminism Edit-A-Thon Events on Newcomers". Social Informatics 10046: 24-40. Springer International Publishing AG. DOI: $10.1007 / 978-3-319-47880-72$

Fuchs, Christian. 2014. Social media: a critical introduction. Sage Publications Ltd. Giles Jim. 2005. “Internet encyclopaedias go head to head." Nature 438: 900-901. 
Glott, Ruediger, Philipp Schmidt, and Rishab Ghosh. 2010. "Wikipedia survey-overview of results." United Nations University: Collaborative Creativity Group.Graham, Mark, Ralph K. Straumann, and Bernie Hogan.2015. "Digital divisions of labor and informational magnetism: Mapping participation in Wikipedia." Annals of the Association of American Geographers, 105(6): 1158-1178.

Haklay, Mordechai. 2010. "How good is volunteered geographical information? A comparative study of OpenStreetMap and Ordnance Survey datasets". Environment and planning. B, Planning \& design 37(4): 682-703.

Erhart, Ed and Aaron Halfaker. 2015. "Wikipedia's very active editor numbers have stabilizeddelve into the data with us." Wikimedia blog. https://blog.wikimedia.org/2015/09/25/wikipedia-editor-numbers/ Accessed October 26, 2017.

Halfaker, Aaron, Stuart R. Geiger, Jonathan Morgan, and John Riedl. 2013. "The rise and decline of an open collaboration system: How Wikipedia's reaction to popularity is causing its decline". American Behavioral Scientist 57(5): 664-688.

Halfaker, Aaron. 2017. "Interpolating Quality Dynamics in Wikipedia and Demonstrating the Keilana Effect". In Proceedings of the 13th International Symposium on Open Collaboration (p. 19). New York: ACM.

Hill, Benjamin Mako, and Aaron Shaw. 2013. "The Wikipedia Gender Gap Revisited: Characterizing Survey Response Bias with Propensity Score Estimation." PLoS ONE 8(6): e65782. https://doi.org/10.1371/journal.pone.0065782

Isaacs, Shafika. 2007. "ICT in education in South Africa." Survey of ICT and Education in Africa: South Africa Country Report.

Jenkins, Henry (2006). “Convergence Culture: Where Old and New Media Collide". New York: New York University Press.

Krieger, Michel, Emily Margaret Stark, and Scott R. Klemmer. 2009. "Coordinating tasks on the commons: designing for personal goals, expertise and serendipity." In Proceedings of the SIGCHI Conference on Human Factors in Computing Systems: 1485-1494. New York: ACM 2009.

Lam, S.T.K., Uduwage, A., Dong, Z., Sen, S., Musicant, D.R., Terveen, L. and Riedl, J., 2011, October. WP: clubhouse?: an exploration of Wikipedia's gender imbalance. In Proceedings of the 7th international symposium on Wikis and open collaboration (pp. 1-10). ACM.

Lavin, Taila. 2016. "A Feminist Edit-a-Thon Seeks to Reshape Wikipedia”. New Yorker, March 11, 2016. Accessed September 18, 2017.

https://www.newyorker.com/tech/elements/a-feminist-edit-a-thon-seeks-to-reshapewikipedia

Loren Terveen, and John Riedl. 2011. “WP: clubhouse?: an exploration of Wikipedia's gender imbalance." In Proceedings of the 7th international symposium on Wikis and open collaboration: 1-10). New York: ACM 2011

Marheineke, Marc, Vivek K. Velamuri, and Kathrin M. Möslein. 2016. "On the importance of boundary objects for virtual collaboration: a review of the literature." Technology Analysis \& Strategic Management 28 (9): 1108-1122.

Messer-Kruse, T. 2012. "The "Undue Weight" of Truth on Wikipedia". The Chronicle. February 12, 2012. Accessed January 5, 2018. http://chronicle.com/article/The-Undue-Weightof-Truth-on/130704/

Morgan, J.T., Gilbert, M., McDonald, D.W. and Zachry, M., 2013, August. Project talk: Coordination work and group membership in WikiProjects. In Proceedings of the 9th International Symposium on Open Collaboration (p. 3). ACM.

Reagle, Joseph, and Lauren Rhue. 2011. "Gender Bias in Wikipedia and Britannica”. International Journal of Communication 5: 1138-1158. Reich, Eugenie Samuel. 2011. "Online reputations: Best face forward”. Published online 11 May 2011. Nature 473, 138-139. doi:10.1038/473138a 
Sugimoto, C.R., Work, S., Larivière, V. and Haustein, S., 2017. Scholarly use of social media and altmetrics: A review of the literature. Journal of the Association for Information Science and Technology, 68(9), pp.2037-2062.

Sapsed, Jonathan, and Ammon Salter. 2004. "Postcards from the edge: Local communities, global programs and boundary objects." Organization Studies 25(9): 1515-1534. Shirky, Clay. 2009. Here comes everybody: How change happens when people come together. London: Penguin

South African Department of Education, Pretoria. 2002. "Revised National Curriculum Statement Grades R-9." Gazette No. 23406, Vol. 433, May 2002. Available at http://www.education.gov.za/Portals/0/CD/GET/doc/economic.pdf?ver=2006-11-21$\underline{095830-000}$

Star, Susan Leigh, and James R. Griesemer. 1989. "Institutional ecology, translations' and boundary objects: Amateurs and professionals in Berkeley's Museum of Vertebrate Zoology, 1907-39". Social studies of science 19(3): 387-420.

Star, Susan Leigh. 2010. "This is not a boundary object: Reflections on the origin of a concept." Science, Technology, \& Human Values 35 (5): 601-617.

Stephens, Monica. 2013. "Gender and the GeoWeb: divisions in the production of user-generated cartographic information." GeoJournal 78 (6): 981-996.

UNESCO. 2015 "Education for all 2000-2015: Achievements and Challenges." EFA Global Monitoring Report 2015. Accessed September 18, 2017. http://unesdoc.unesco.org/images/0023/002322/232205e.pdf

Warncke-Wang, Morten, Vladislav R. Ayukaev, Brent Hecht, and Loren Terveen. 2015. "The success and failure of Quality Improvement Projects in peer production communities". In CSCW 2015 - Proceedings of the 2015 ACM International Conference on Computer-Supported Cooperative Work and Social Computing: 743-756. Association for Computing Machinery, Inc. DOI: $10.1145 / 2675133.2675241$

Wikipedia. 2017. "Wikipedia:Featured Article Criteria”. Last modified February 19, 2017. https://en.wikipedia.org/wiki/Wikipedia:Featured_article_criteria

World Economic Forum (WEF). 2013. "Global Competitiveness Report 2016-2017”. Accessed September 18, 2017. http://www3.weforum.org/docs/WEF_GlobalCompetitivenessReport_2012-13.pdf 